\title{
Visual masking by a patterned stimulus and recovery of observer performance
}

\author{
DEAN G. PURCELL \\ Oakland University, Rochester, Michigan 48063 \\ and \\ ALAN L. STEWART \\ New York University, New York, New York 10003
}

\begin{abstract}
A forced-choice visual acuity task was used to assess visual backward masking and recovery in two experiments. In the masking conditions, the target was closely followed in time by a patterned stimulus. In the recovery condition, the patterned masking stimulus was followed, in turn, by a homogeneous flash of light. The homogeneous flash was found to enhance observer performance. This enhancement held under both dark adaptation and light adaptation as well as when observers were given immediate feedback about their performance.
\end{abstract}

The report of a visual stimulus may be interfered with if the onset of the target stimulus is followed closely in time by a second visual stimulus. Because the second stimulus sometimes interferes with the perception of the target, it is called a masking stimulus, and is referred to as Mask 1 in this paper. If an additional stimulus is added to this sequence, so that the onset of Mask 1 is followed by the onset of a third stimulus, target report may improve. This third stimulus will be referred to somewhat arbitrarily as Mask 2. We have loosely referred to the improvement of the observer's performance with the addition of Mask 2 to the stimulus train as an indication of the recovery of the target; others have referred to this result as disinhibition of an inhibited target. The means by which the perception of a target is suppressed by a masking stimulus, however, are less well understood than the terms recovery or disinhibition might suggest. Therefore, a better convention might be to talk only about the recovery of the observer's ability to make a more accurate judgment of a target. In any event, an improvement in dealing with what would otherwise be masked targets has been found for letters of the alphabet (Dember \& Purcell, 1967; Long \& Gribben, 1971) and for disk targets (Purcell \& Dember, 1968; Robinson, 1966, 1968; Schiller \& Greenfield, 1969; Stewart, Purcell, \& Dember, 1968).

In all of the studies listed above, the border of the stimulus used as Mask 1 did not fall directly on the portions of the retina stimulated by the target figure. As a result, each experiment might be classified methodologically as either a metacontrast or a

Requests for reprints should be sent to Dean G. Purcell, Department of Psychology, Oakland University, Rochester, Michigan 48063 . Research was partially supported by a grant from the Rehabilitation Services Administration to the second author. masking-by-flash experiment. Therefore, it is of some interest simply to discover if recovery is restricted to those paradigms or if recovery may also take place when the mask (Mask 1) is a complex pattern of overlapping forms, each element being about the size of the target elements, like the masks used in many information processing studies.

A series of experiments published recently by Turvey (1973) offers the only instance of recovery with patterned masking stimuli. In these experiments, recovery was reported when Mask 1 was a patterned stimulus and Mask 2 was either a collection of patterned elements, each element being significantly smaller than the elements making up the target, or a homogeneous flash of light. Observers were not required to make a forced-choice identification of the target, however. Instead, the observers reported how distinctly they were able to see the target under the masking and recovery conditions. Consequently, Turvey's experiments on recovery are not as convincing as they might be, although we found the impression of improved legibility of the target to be compelling when we viewed similar conditions.

Compelling as Turvey's demonstration may be when one observes it in person, recent criticisms have centered on other aspects of recovery experiments. It is not disputed that observer performance can sometimes be improved by adding a third stimulus to the masking sequence; rather, the claim is made that recovery occurs only when the observer has first adopted a brightness criterion inappropriate to target detection under masking conditions (Barry \& Dick, 1972). According to this position, whenever recovery occurs, the first masking stimulus has served only to change the target's brightness without effecting any change in the information about the target's outline. In those situations in which the target has a 
phenomenal representation, we have called this a reversal of the target's brightness (Purcell \& Dember, 1968; Stewart, Purcell, \& Dember, 1968). The second masking stimulus in recovery paradigms, then, induces a rereversal of the brightness changes effected by the first mask. As adapted by Barry and Dick, this brightness interpretation holds that recovery can take place only when the target, followed closely in time by a second stimulus (Mask 1), comes to have an altered but nonetheless characteristic phenomenal representation. From this reasoning, it follows that in those masking situations in which the addition of a second mask will result in recovery, an observer can correctly report the target's presence if he is made aware of brightness changes associated with each SOA, and thus is allowed to modify his criterion appropriately from one condition to the next.

Previous papers have dealt with the role which an observer's criterion may play in masking experiments when target detection is based upon explicit brightness judgments (Purcell \& Dember, 1968; Purcell, Stewart, \& Dember, 1968; Stewart, Purcell, $\&$ Dember, 1968). The present paper describes a more stringent example of recovery, and demonstrates recovery in a masking-by-pattern paradigm, as well. In this example, a single masking stimulus apparently reduces observer performance by decreasing the amount of information the observer has about a target's outline, while a sequence of two masking stimuli results in improved observer performance. Consequently, it represents an instance of the recovery phenomenon in which the target does not have an identifiable representation when followed only by a single masking stimulus.

In the two experiments reported in this paper, the masking stimulus (Mask 1) was a complex pattern and Mask 2 was a homogeneous flash of light. The target stimuli consisted of two annuli, one of which had a portion missing. In both experiments, observers made a forced-choice decision as to which annulus was presented on any given trial. In the second of the two experiments, observers were given immediate feedback after each response. Immediate feedback would bring any phenomenal change in the target to the observers' attention, causing them to adopt an appropriate criterion for reporting the occurrence of the target. In this way, the objections raised by Barry and Dick (1972) to other demonstrations of recovery would not apply to these data. Moreover, by using an acuity task, and by obtaining a forced decision from the observer, as well, these experiments, along with Turvey's experiments, serve to further link the recovery phenomenon with the masking paradigms used in visual information processing experiments.

\section{EXPERIMENT I}

\section{Method}

Stimuli. The target stimulus was one of two black-on-white annuli, eacin having an outer diameter of $.53 \mathrm{deg}$ and a s:rp width of $.07 \mathrm{deg}$. The annulus designated as the target had a break positioned at its top, causing it to resemble a letter $C$ rotate -9 ) deg upward. The size of the break in the target annulus was adjusted for each observer so as to yield $50 \%$ target detection at a 20 -msec separation between target and Mask 1 onsets. Accordingly, two observers were tested using an annulus with a break of .13 deg, and three were tested with breaks of . $20 \mathrm{deg}$.

The target field was square and subtended $3 \mathrm{deg}$ on each side. The center of the field was positioned $.56 \mathrm{deg}$ to the right of a fixation point located directly ahead of the observer. The target luminance was approximately $6.36 \mathrm{~cd} / \mathrm{m}^{2}$; the white portions of the target field were set at $63.66 \mathrm{~cd} / \mathrm{m}^{2}$. The Mask 1 field was the same size as the target field, was positioned so as to appear superimposed on it during simultaneous exposures, and contained a pattern made up of overlapping rows and columns of the black-on-white letters $\mathrm{N}$ and $O$. Each letter of the masking pattern was approximately the same size as the target stimulus. The pattern itself was $3 \mathrm{deg}$ wide and $2.55 \mathrm{deg}$ high and was centered within the Mask 1 field. The luminance of the white portions of the patterned mask was constant at $63.66 \mathrm{~cd} / \mathrm{m}^{2}$.

The second mask, Mask 2, consisted of a 3-deg-square white field at a luminance of $63.66 \mathrm{~cd} / \mathrm{m}^{2}$. This field was centered on the target and Mask 1 fields. Target, Mask 1, and Mask 2 durations were constant at 20 msec. All stimuli were presented via an Iconix tachistoscope.

Procedure. A masking trial consisted of the presentation of the target field, followed after appropriate intervals by Mask 1 and Mask 2. On each trial, one of the stimuli, either the broken or the unbroken annulus, was presented according to a random sequence. After each trial, the observers responded "yes," to indicate that they had seen the break within the wall of the annulus, or "no," to indicate that they had seen no gap.

Convent:onally, stimulus onset asynchrony values are nega:ive if mask ons:t follow's target onset in time. The stimulus onset asynchron: of target and Mask 1, and between Mask 1 and Mask 2, w ill be ref zrred to as negative $S O A 1$ and negative $\left.S^{\prime}\right) A 2$, respectively. The percent correct target detection was determined for each observer under negative values of SOA 1 at 20,40 . and $60 \mathrm{msec}$. In the recovery condition, the negative SOA 2 was constant at $20 \mathrm{msec}$. In the masking condition, Mask 2 followed the onset of Mask I by $1 \mathrm{sec}$, thereby keeping the adaptation between masking and recovery conditions constant. The intertrial interval was $10 \mathrm{sec}$.

The fixation field consisted of four peripherally located red pinpoints of light, arranged in a diamond pattern, with a central fixation point. The observers were instructed to initiate each trial by pressing a telegraph key, once they had carefully fixated the central fixation point. All viewing was monocular with the right eye. Observers were adapted for $10 \mathrm{~min}$ before each experimental session.

Fifty trials were collected under each experimental condition. These conditions were combined into a 2 by 3 repeated measures analysis of variance design, with data collected in random blocks of 10 trials.

\section{Results}

The results of Experiment $I$ are shown in Table 1. There was no statistically significant difference among the three values of SOA 1. A comparison between masking and recovery conditions, however, demon- 
strated that target detection was higher for the recovery condition; this difference was statistically significant, $F(1,4)=71.08, p<.005$, demonstrating that both masking and recovery of contour information can occur when the masking stimulus is a complex pattern of overlapping figures.

\section{EXPERIMENT II}

\section{Method}

The annuli were those used in Experiment I. The size of the break in the annulus designated as the target was set at $.07 \mathrm{deg}$ for three observers and $.13 \mathrm{deg}$ for the fourth observer. The dimensions and configurations of the fixation, target, Mask 1, and Mask 2 fields were identical to those in Experiment I.

The surround luminance of the target field and the luminance of the white portions of Mask 1 were constant at $63.66 \mathrm{~cd} / \mathrm{m}^{2}$. The luminance of Mask 2 was set at $76.39 \mathrm{~cd} / \mathrm{m}^{2}$. The durations of target, Mask 1, and Mask 2 were, respectively, 15, 20, and $50 \mathrm{msec}$. The fixation field was continuously illuminated at $39.46 \mathrm{~cd} / \mathrm{m}^{2}$.

Target and Mask 1 onset were separated from each other by negative SOA 1 values of $20,30,40$, and $50 \mathrm{msec}$. In the recovery condition, negative SOA 2 was constant at $20 \mathrm{msec}$. Because there was a continuously illuminated fixation field, the adaptation effects of Mask 2 were no longer an important consideration; therefore, in the masking conditions, Mask 2 was no longer presented. Observers were adapted to the fixation field luminance for $10 \mathrm{~min}$ before each experimental session. Data were collected as in Experiment I, except that the observers were given feedback after each trial.

\section{Results}

The results of Experiment II are shown in Table 1. There was a statistically significant difference between recovery and masking conditions, $F(1,3)=16.22$, $\mathrm{p}<.05$, as well as a statistically significant effect of varying SOA $1, F(3,9)=20.88, p<.001$. The masking conditions also interacted with SOA 1 , and proved to be statistically significant, with SOA 1 producing smaller differences in percent correct detection in the recovery condition than in the masking condition, $F(3,9)=6.64, p<.025$. Experiment II, then, indicates that both masking and recovery were stable phenomena even when observers were given feedback immediately after each response. In addition, recovery was obtainable under conditions of light adaptation.

\section{GENERAL DISCUSSION}

We have suggested previously that some of the conditions under which recovery can occur are such that, even after interacting with the masking stimulus, the target still comes to have a distinct phenomenal representation-albeit one completely unsuspected by the observer (Purcell \& Dember, 1968; Stewart, Purcell, \& Dember, 1968). The data presented in this paper suggest that this phenomenal representation is not iecessary for recovery, however, and, indeed, that there may be another form of the recovery phenomenon. Specifically, there seem to be circumstances under which the target has no distinct phenomenal representation once it has interacted with a single masking stimulus, but, nonetheless, becomes phenomenally distinct as soon as an appropriate third stimulus is added to the train of target and masking stimuli.
Table 1

Mean Percentage Target Detection Under Conditions of Masking and Recovery

Negative SOA from Target Onset to Mask 1 Onset
20

$\begin{array}{ll}\text { Masking } & 54.8 \\ \text { Recovery } & 75.2\end{array}$

Masking

Recovery
54.8
75.2

54.5
30

Experiment I
61.6

61.6
76.4

Experiment II

$\begin{array}{lll}64.0 & 85.0 & 93.5\end{array}$

Masking with a patterned masking stimulus would seem to be one of these latter circumstances. Suppose, for example, that a pattern of reversals produced by stimulation of the same areas of the retina, first by the black portions of the target and then by the more intensely illuminated portions of the masking pattern, resulted in a phenomenally distinct pattern of fragmented letter parts. Suppose, in addition, that the second mask had then induced a second reversal of this pattern. Even then, correctly identifying the rereversals of this fragmented letter should have remained as difficult a task as identifying the originally fragmented letter. Previous experiments on recovery with patterned masking stimuli, by omitting immediate feedback to observers after each masking trial, had left open the possibility that the target remained phenomenally distinct after the offset of the patterned masking stimulus (Mask 1) and that, had his attention been drawn to it, the observer could have correctly reported the target. With the use of immediate feedback in our experiments, on the other hand, the observer was free to modify his response criterion so as to best take advantage of all the information available to him at each SOA value. The fact that differences in correct report between the masking and recovery conditions still occurred indicates that an absolute improvement in the observer's performance was a result of including a third stimulus as part of the train of target and mask stimuli. In this instance, then, no distinct phenomenal trace of the target would seem to be available to the observer before the onset of the second masking stimulus (Mask 2). These remarks would refer, of course, only to those values of SOA under which masking occurs.

As well as further documenting what might well be a second form of the recovery phenomenon, these experiments have an additional theoretical importance. Along with the experiments of Dember and Purcell (1967), Long and Gribben (1971), and Turvey (1973), the present experiments demonstrate both masking and recovery with visual targets which were reported on the basis of their shape rather than brightness. As such, each of these studies would indicate that the processing of visual information is not terminated by the onset of the first mask; otherwise, once the mask had onset, no further information would be available to the observer, and no way for performance to be enhanced by a second masking stimulus would exist.

If, in general, it is the continuing activity of the mask, over a short but definite span of time after the offset of the masking stimulus, which serves to eliminate any usable trace of the target, recovery can then be understood as another expression of visual masking itself. All that is required for recovery to occur is that the activity of the mask be rendered ineffective before it has removed all trace of the target.

While an interruption explanation of masking would hold that recovery phenomena are unrelated to most forms of visual masking, alternative explanations of masking make the grouping of recovery with other masking phenomena more plausible. In effect, it is the interference with the mask by an additional stimulus which allows for better performance of the observer under some multiple stimulus masking paradigms. It remains a moot point whether this interference is an expression of inhibition (Weisstein, 1968) or of some combination of summation and inhibition (Stewart \& Purcell, 
1974; Turvey, 1973). The important consequence of this approach, however, is that if it is the activity of the mask, distributed over time, which allows for a parsimonious explanation of recovery, the need for an interruption explanation of visual masking in more conventional masking paradigms becomes otiose.

\section{REFERENCES}

BarRY, S. H., \& Dick, A. O. On the recovery of masked targets. Perception \& Psychophysics, 1972, 12, 117-120.

Dember, W. N., \& Purcell, D. G. Recovery of masked visual targets by inhibition of the masking stimulus. Science, 1967, 157, 1335-1336.

LoNG, N. R., \& GribBen, J. A. The recovery of a visually masked target. Perception \& Psychophysics, 1971, 10, 197-200.

Purcell, D. G., \& Dember, W. N. The relation of phenomenal brightness reversal and re-reversal to backward masking and recovery. Perception \& Psychophysics, 1968, 3, 290-292.

Purcell, D. G., Stew art, A. L., \& Dember, W. N. Spatial effectiveness of the mask: Lateral inhibition in visual backward masking. Perception \& Psychophysics, 1968, 4, 344-346.
Robinson, D. N. Disinhibition of visually masked stimuli. Science, 1966, 154, 157-158.

Robinson, D. N. Visual disinhibition with binocular and interocular presentations. Journal of the Optical Society of America, 1968, 58, 254-257.

Schiller, P. H., \& Greenfield, A. Visual masking and the recovery phenomenon. Perception \& Psychophysics, 1969, 6, 182-184.

Stewart, A. L., \& Purcell, D. G. Visual backward masking by a flash of light: A study of U-shaped detection functions. Journal of Experimental Psychology, 1974, 103, 553-566.

Stew art, A. L., Purcell, D. G., \& Dember, W. N. Masking and recovery of target brightness. Proceedings of the American Psychological Association, 1968, 3, 109-110.

TuRveY, M. T. On peripheral and central processes in vision: Inferences from an information-processing analysis of masking with patterned stimuli. Psychological Review, 1973, 80, 1-52.

Weisstein, N. A Rashevsky-Landahl neural net: Simulation of metacontrast. Psychological Review, 1968, 75, 494-521.

(Received for publication June 26, 1975.) 